\title{
Curative effect of HFIO on liver and peritoneal metastasis mediated by host antitumor immunity
}

This article was published in the following Dove Press journal:

Oncolytic Virotherapy

13 March 2017

Number of times this article has been viewed

\author{
Yoshihiro Hotta' \\ Hideki Kasuya ${ }^{2}$ \\ Itzel Bustos ${ }^{2}$ \\ Yoshinori $\mathrm{Naoe}^{2}$ \\ Toru Ichinose ${ }^{2}$ \\ Maki Tanaka ${ }^{3}$ \\ Yasuhiro Kodera' \\ 'Department of Surgery II, ${ }^{2}$ Cancer \\ Immune Therapy Research Center, \\ Nagoya University Graduate School \\ of Medicine, Nagoya, ${ }^{3}$ Takara Bio Inc., \\ Otsu, Japan
}

Background: HF10 is a highly attenuated type 1 herpes simplex virus (HSV) with proven effective oncolytic effect. Previous investigations have demonstrated that colon cancer mice model treated with HF10 not only had better survival but were also resistant to the reimplantation of the antitumor effect mediated by host antitumor immunity. Importantly, it has also been noted that in mice with antitumors implanted on both sides of the back, an injection of HF 10 on only one side strongly restrains not only the injected antitumor but also the non-injected ones. Materials and methods: MC26 colon cancer cells were injected subcutaneously into the back, spleen, and intraperitoneal region of metastasis model mice. Antitumor volume and survival rate were monitored. To measure cytotoxic T lymphocytes (CTL) cytotoxicity against MC26, lymphocytes were extracted from the spleens of the peritoneal metastasis model mice as well as from the thymus of the liver metastasis model mice. The expression of interferon gamma was examined by enzyme-linked immunospot assay. Samples from the liver metastasis model mice were subjected to polymerase chain reaction to quantify the level of HSV genomes.

Results: HF10 was injected only on the back antitumor; however, a antitumor-suppressor effect was observed against liver and peritoneal metastases. When HF10 genome was measured, we observed lower genome on liver metastases compared to back antitumor genome quantity. CTL activity against MC26 was also observed. These results indicate that local administration of HF10 exerts a curative effect on systemic disease, mediated by host antitumor immunity.

Conclusion: HF10 local administration stimulates antitumor immunity to recognize antitumorspecific antigen, which then improves systemic disease. Metastatic antitumors lysis, on the other hand, appears to be mediated by the host immune system, rather than by virus-mediated direct oncolysis.

Keywords: herpes oncolytic virus, antitumor metastasis, immune response, T-cell-mediated cytotoxicity

\section{Introduction}

Stomach cancer, which some years ago was the leading cause of cancer mortality in Japan, is now the third most common cause of cancer-related deaths in Japan, whereas colon cancer is the first cause of morbidity and the second leading cause of cancer mortality in Japan as of 2015. 1,2-5 Many patients with advanced colon cancer cannot undergo curative surgery due to liver or peritoneal metastasis. Taking this into account, we investigated the effect of HF10 in colon cancer and observed the host immune response in the presence of oncolytic virus. For this purpose, we used an immunecompetent mice model harboring colon cancer.

Oncolytic viruses are predicted to be an effective tool against advanced cancer, and they have extensively been tested in clinical studies. Many studies have investigated 
a wide range of oncolytic viruses and have reported their effectiveness: a Phase I clinical trial using intravenous administration of PV701, an oncolytic Newcastle disease virus; ${ }^{6}$ experimental therapy of human glioma using a genetically engineered mutant of herpes virus $;{ }^{7}$ systemic cancer therapy using a antitumor-selective mutant of vaccinia virus, ${ }^{8}$ a Phase II clinical trial of intratumoral administration of ONYX-015 against refractory head and neck cancer; ${ }^{9}$ and intraperitoneal therapy of ovarian cancer using an engineered measles virus. ${ }^{10}$ All these studies have reported the effectiveness and safety of their oncolytic viruses against various cancers. Oncolytic viruses have been receiving increasing attention year by year as new biological anticancer therapies.

In October 2015, an oncolytic type 1 herpes simplex virus (HSV-1), talimogene laherparepvec (T-VEC, Imlygic ${ }^{\mathrm{TM}}$, Amgen, Cambridge, UK), ${ }^{11-13}$ was approved for clinical use by the US Food and Drug Administration. Therefore, oncolytic viral therapy is no longer only the dream of a few physician-scientists, rather it has become a clinically approved therapy. In the near future, oncolytic viruses will be deployed as novel biological agents against various advanced cancer.

HF10 is a spontaneously occurring HSV-1 mutant that lacks functional expression of UL43, UL49.5, UL55, UL56, and latency-associated transcripts. ${ }^{14}$ Deletion of UL56 is associated with a lack of neuroinvasiveness and is believed to attenuate its pathogenicity and neurovirulence relative to wild-type HSV-1. Normal cells produce interferon, which activates protective antiviral signaling in the surrounding cells and inhibits the replication pathway, allowing the surrounding uninfected cells to survive. On the other hand, cancer cells support viral replication due to their weak response to interferon and overexpression of replication pathways such as Ras at antiviral signaling pathways including protein kinase receptor (PKR). Owing to this difference between normal cells and cancer cells, HF10 is antitumor-selective. Our laboratory has reported the curative effects of HF10 in several malignant antitumors. ${ }^{15-18}$

Nonresectable cases of malignant abdominal antitumors have been the targets of many clinical studies. Unfortunately, at the time of diagnosis, liver metastasis or peritoneal metastasis already existed in most of the cases due to rapid tumor prognosis. Mice treated with HF10 become resistant to reimplantation of the antitumor. ${ }^{19,20}$ In addition, in mice implanted with antitumors on both sides of the back, an injection of HF10 into one side of the antitumor strongly suppresses the non-injected antitumor. ${ }^{21}$ The specific contribution of host immunity to these phenomena is not yet totally understood. Therefore, we examined the curative effect of local administration of HF10, mediated by antitumor immunity, on systemic metastasis.

\section{Materials and methods Cells and viruses}

Vero, an African Green monkey kidney cell line, was obtained from the American Type Culture Collection (Manassas, VA, USA) and used for virus propagation and virus tittering. Plaque-forming unit (PFU) of HF10 was determined on Vero cells with agarose coating. MC26, a mouse colon carcinoma cell line, was obtained from the National Cancer Institute Antitumor Repository (Frederick, MD, USA). Vero and MC26 cells were maintained in Dulbecco's modified Eagle's medium (DMEM) with 10\% fetal bovine serum (FBS) and 1\% penicillin/streptomycin. $\mathrm{HF} 10$ was stored with DMEM without FBS at $-80^{\circ} \mathrm{C}$ and incubated at $37^{\circ} \mathrm{C}$ just before use.

\section{Viral cytotoxic assay}

MC26 cells were plated onto 96-well plates (4,000 cells/ well) and cultured for $36 \mathrm{~h}$. HF 10 was added at multiplicity of infection (MOI) values ranging from 0.001 to 10 , and the infected cells were incubated for 3 days. The number of surviving cells was quantified by colorimetric MTT assay (Sigma-Aldrich, St Louis, MO, USA), and cell survival percentage was calculated by comparison with control (mockinfected) cells. Tests were performed in quadruplicate.

\section{Animal studies}

All experiments were approved by Nagoya University Animal Ethics Committee and conducted in accordance with the general guidelines issued by the Nagoya University Animal Center. BALB/c mice were obtained from Central Institute for Experimental Animals (CLEA) Japan (Tokyo, Japan). Mice were divided into four groups, and each group included 10 mice: liver metastasis model, treated with or without HF10, and peritoneal metastasis model, treated with or without HF10. Liver metastasis models were generated by injecting a single-cell suspension of $10^{6} \mathrm{MC} 26$ cells in $100 \mu \mathrm{L}$ Hank's buffered salt solution (HBSS) into the spleen of BALB/c mice. Peritoneal metastasis models were generated by subcutaneously injecting a single-cell suspension of $10^{6} \mathrm{MC} 26$ cells in $1,000 \mu \mathrm{L}$ HBSS into the intraperitoneal region of $\mathrm{BALB} / \mathrm{c}$ mice. All models were also injected with MC26 cells subcutaneously on their backs. HF10 $\left(1 \times 10^{7}\right.$ PFU) was injected into the subcutaneous antitumors on day 10,17 , and 24 after antitumor implantation. After antitumor injection, we monitored subcutaneous antitumor size and 
the duration of survival ( $\mathrm{n}=10$ /group). The level of HF10 genome was detected by polymerase chain reaction (PCR) in samples derived from liver tissue and subcutaneous antitumors of the liver metastasis model.

\section{Lymphocyte preparation}

We assembled similar groups of mice for extraction of whole subsets of lymphocytes ( $n=5$ /group). Spleens were extracted from the peritoneal metastasis models on day 17 , and cytotoxic T lymphocytes (CTL) activity against MC26 was measured by MTT assay. In addition, thymuses were extracted from liver metastasis models on day 17. Lymphocytes were harvested and further purified by Lympholyte-M Cell Separation Media (CEDARLANE, Burlington, ON, Canada). CTL activity against MC26 was measured by MTT assay and interferon gamma (IFN- $\gamma$ ) expression was monitored by enzyme-linked immunospot (ELISPOT) assay.

\section{PCR analysis}

PCR amplification of HF10-specific sequences was performed to investigate the biodistribution of HF10 following intratumor administration. DNA from mouse liver and subcutaneous antitumor tissues was extracted using PureLink Genomic DNA Mini kit (Life Technologies, Carlsbad, CA, USA). Quantitative PCR from $50 \mathrm{ng}$ of total DNA was carried out with StepOnePlus Real-Time PCR System (Applied Biosystems, Carlsbad, CA, USA) using TaqMan Fast Advanced Master Mix (Applied Biosystems). The UL1 region targeting primers used were as follows: sense 5'-ACGCCGCCACAAAAAGC-3', antisense 5'-TCCGTCGAGGCATCATTAGAT-3', and probe 6-carboxyfluorescein/5-carboxytetramethylrhodamine (FAM/TAMRA) 5'-CCGACGACCCCATTCCCGG-3'. For standard HF10 DNA, HF10 UL1 region was amplified and cloned into pCR2.1-TOPO TA vector (Life Technologies). HF10 DNA copy number was determined with standard comparison curve.

\section{Histochemical analysis by HFIO immunostaining}

We extracted liver and subcutaneous antitumor from liver metastasis models on days 1 and 7 after HF10 therapy and froze it rapidly in optimal cutting temperature compound in liquid nitrogen $(n=3 /$ each). Tissues were immunohistochemically stained with anti-HSV antibody (1/100 dilution; Novus Biologicals, San Diego, CA, USA). Histofine ${ }^{\circledR}$ biotinylated antirabbit immunoglobulin $\mathrm{G}$ secondary antibody kit (Nichirei Biosciences Inc., Tokyo, Japan) was used according to the operation instructions. Histofine horseradish peroxidase-3,3-diaminobenzidine ready-to-use kit (Nichirei Biosciences Inc.) was used as the chromogenic agent. The HSV-staining spots were counted in one field at $400 \times$ magnification.

\section{Lymphocytic cytotoxic assay}

Spleens were collected from peritoneal metastasis models, and thymus was collected from liver metastasis models. Whole subsets of lymphocytes were extracted from both tissues. MC26 cells were plated onto 96-well plates at 4,000 cells/well for $36 \mathrm{~h}$. Whole subsets of lymphocytes were added $\left(4 \times 10^{4}\right.$ or $4 \times 10^{5}$ cells/well) and incubated for 24 , 48 , and $72 \mathrm{~h}$. The number of surviving cells was quantified by colorimetric MTT assay (Sigma-Aldrich) ( $n=5 /$ group). Tests were performed in quadruplicate.

\section{IFN- $\gamma$ ELISPOT assay}

We extracted whole subsets of lymphocytes from freshly isolated mouse splenocytes and thymus, adjusted cell concentration to $2 \times 10^{5}$ cells $/ \mathrm{mL}$, and plated them at $1 \times 10^{5}$ cells/ well in 96-well ELISPOT plates (BD Bioscience, Franklin Lakes, NJ, USA) coated with antimouse IFN- $\gamma$ antibody at $50 \mu \mathrm{L} /$ well. The lymphocytes were stimulated with $1 \times 10^{3} /$ $\mathrm{mL} \mathrm{MC26}$ cells. ELISPOT plates were incubated for $20 \mathrm{~h}$ at $37^{\circ} \mathrm{C}$ under $5 \% \mathrm{CO}_{2}$ and then read by visual observation.

\section{Statistical analysis}

Continuous variables were compared by Student's $t$-test. Survival data were analyzed using Kaplan-Meier curves and comparisons were evaluated by the log-rank test. Differences were considered statistically significant when $P$ values were less than 0.05 . All analyses were conducted using JUMP 8 software (SAS Institute Inc., Cary, NC, USA).

\section{Results}

\section{Cytotoxic effect of HFIO against MC26 in vitro}

We examined the cytotoxic effect of HF10 in the mouse colon carcinoma cell line MC26. We infected the cultured cells with HF10 at MOI values ranging from 0.001 to 10 and then compared the survival of infected vs uninfected cells by MTT assay. The results revealed that about $90 \%$ of the cells survived at MOI 0.001 , whereas fewer than $5 \%$ of cells survived at MOI 10. Therefore, HF10 exerted high and dose-dependent cytotoxicity against MC26 (Figure 1). 


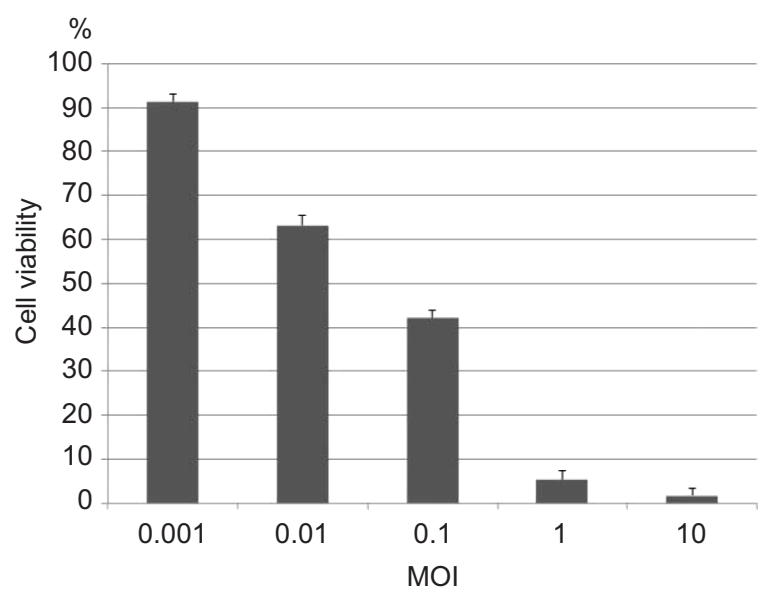

Figure I HFIO exhibited a strong dose-dependent cytotoxic effect against MC26. The mouse colon carcinoma cell line MC26 was infected with HFIO at several MOI values, and surviving cells were quantified 6 days after infection. Survival rates of each experimental group were compared with that of the control group (noninfected). Abbreviation: MOI, multiplicity of infection.

\section{HFI0 prolonged the survival of MC26-bearing mice}

As HF10 showed strong cytotoxic effect against MC26 in vitro, antitumor activity of HF10 in antitumor metastasis models (liver and peritoneal metastasis) was investigated. After administration of HF10 into a subcutaneous antitumor, we measured the antitumor size and monitored the survival of mice. HF10 treatment significantly suppressed the growth of subcutaneous antitumor in both metastasis models (Figure 2A). Furthermore, HF10 prolonged the survival of mice in the metastasis models (Figure 2A). Mice treated with HF10 did not exhibit neurologic symptoms on gait or rapid weight loss after HF10 injection (data not shown).

\section{HFIO reduced peritoneal and liver metastasis}

Since HF10 treatment prolonged mice survival in the metastasis models, we examined whether HF10 reduced antitumor metastasis in the models. Mice were treated with HF10 in subcutaneous antitumor and sacrificed on day 17 after antitumor implantation. In the peritoneal metastasis model, mice treated with $1 \times 10^{7} \mathrm{PFU}$ HF10 in the subcutaneous antitumor had few abdominal antitumor nodules, whereas untreated mice had numerous abdominal antitumor nodules (Figure 2B). Similarly, in the liver metastasis model, untreated mice had numerous antitumor nodules, whereas HF10-treated mice were almost normal, and their liver's weight was significantly lower than that of the untreated mice (Figure 2C). In addition, the spleen's weight of the treated liver metastasis model mice was considerably lower than that of the untreated mice.

\section{HFIO DNA concentration was higher in primary antitumors}

Intratumor administration of HF10 showed strong antitumor effect in subcutaneous antitumor and significantly reduced liver and peritoneal metastasis. We then searched for the possibility of direct antitumor effect of HF10 in affected liver and peritoneal metastasis. After HF10 administration into subcutaneous antitumors, on days 1 and 7 , we extracted DNA from the liver and subcutaneous antitumor tissues of mice and then examined the biodistribution of HSV-1 by PCR. HSV-1 DNA was detected in significantly higher concentrations in subcutaneous antitumor tissue compared to liver tissue on days 1 and 7 (Figure 3A). Similarly, we performed histochemical staining of liver tissue from the liver metastasis group on days 1 and 7 after virus injection. At both time points, liver tissue contained significantly fewer HSV-1-immune-positive spots compared to subcutaneous antitumor tissue (Figure 3B). Together, the results of PCR and histochemical staining demonstrated that the curative effect on liver metastasis was not simply a direct result of HF10 oncolytic effect.

\section{Lymphocytic cytotoxic assay}

As the curative effect on liver metastasis was not due to HF10 oncolysis, the effect conceivably involved the host immune response. To confirm this theory, we performed cytotoxicity assays using the extracted whole subsets of lymphocytes. The lymphocytes from the HF10-injected group were more cytotoxic than that of the control group, with significant difference at $48 \mathrm{~h}$, even at a low relative ratio (10:1) of lymphocytes to MC26 cells (Figure 4). Next, we examined IFN- $\gamma$ production from whole subsets of lymphocytes in the HF10injected mice by ELISPOT assay. After stimulation with MC26, the ELISPOT assay revealed that the lymphocytes of the treated group contained significantly more expression spots compared to the control group (Figure 5). These results demonstrated that lymphocytes from the HF10-injected mice had strong cytotoxic activity as well as high IFN- $\gamma$ production.

\section{Discussion}

The effects of HF10 have been studied in various types of malignant antitumors, including breast, pancreas, colon, and ovarian cancers. ${ }^{13,16,21,22}$ Since 2003, we have been carrying out Phase I clinical trials of HF10 against recurrent breast cancer and advanced head and neck cancer, ${ }^{23-25}$ and since 2005, we have been applying HF10 to the treatment of nonresectable pancreatic cancer. ${ }^{14,22}$ However, the targets of these clinical studies were advanced nonresectable cases, 
A
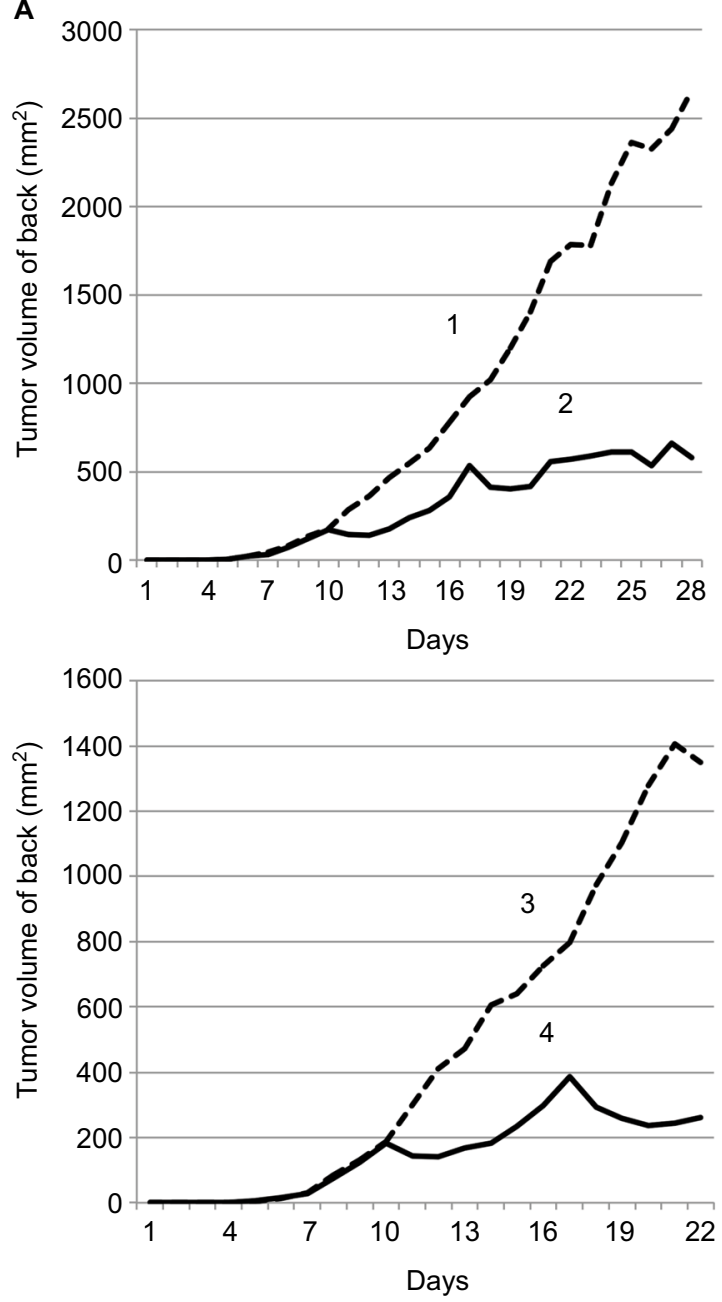

B

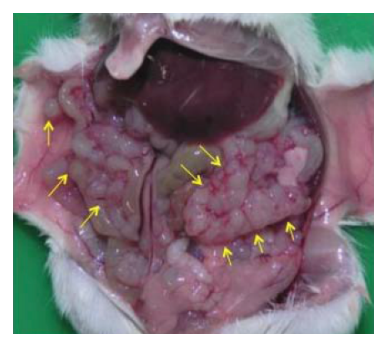

Untreated group

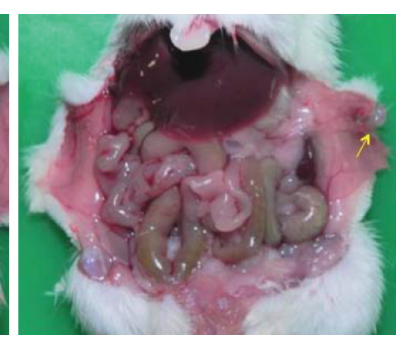

Treated group
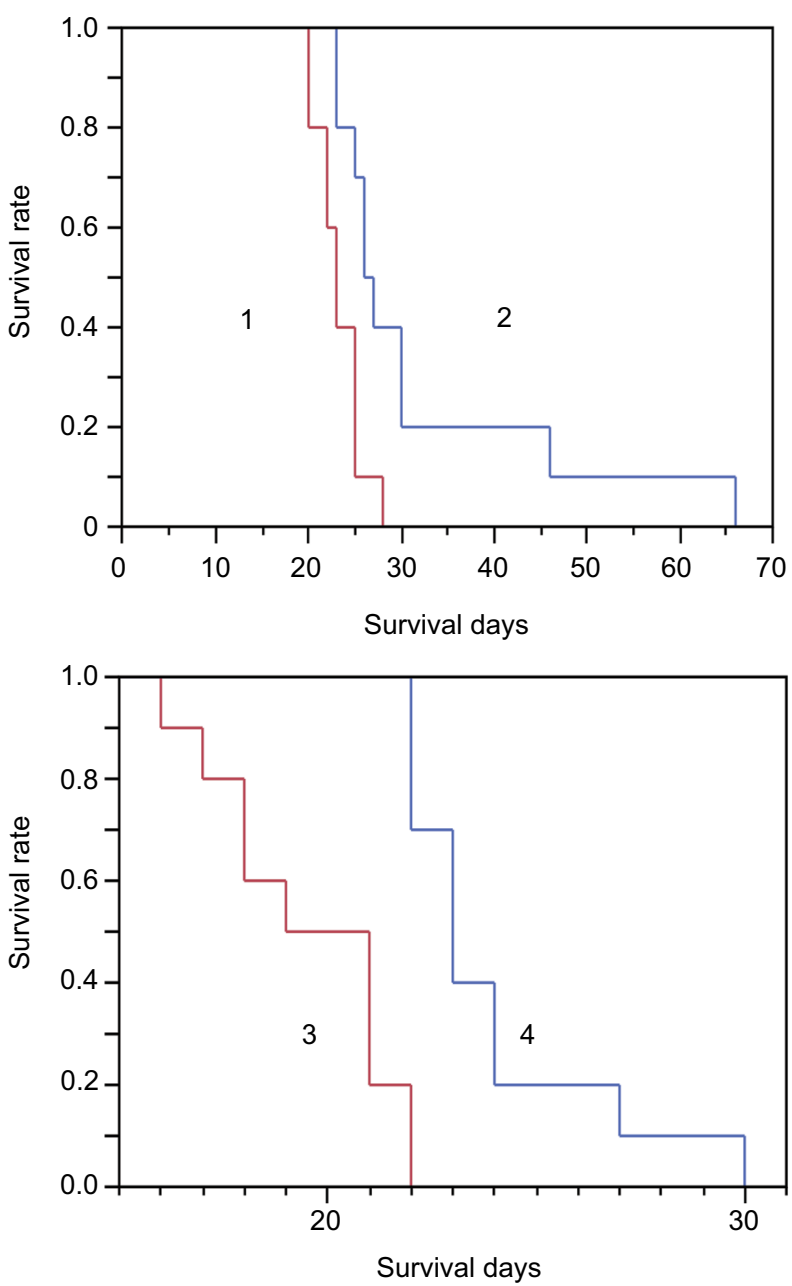

C

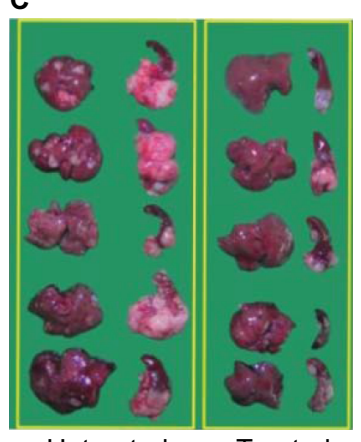

\begin{tabular}{|l|c|c|}
\hline Group & $\begin{array}{c}\text { Liver weight } \\
(\mathrm{g}) \pm \mathrm{SD}\end{array}$ & $\begin{array}{c}\text { Spleen weight } \\
(\mathrm{g}) \pm \mathrm{SD}\end{array}$ \\
\hline Untreated & $1.89 \pm 0.40$ & $1.97 \pm 0.76$ \\
\hline Treated & $1.52 \pm 0.12^{*}$ & $0.63 \pm 0.31^{*}$ \\
\hline
\end{tabular}

${ }^{*} P<0.05$

compared to untreated group

Untreated
Treated

Figure 2 (A) HFI0 prolonged the survival of metastasis model mice. We established four groups and each group included 10 mice. I, liver metastasis, control group; 2, liver metastasis, HFI0-treated group; 3, peritoneal metastasis, control group; 4, peritoneal metastasis, HFI0-treated group. $\mathrm{HFIO}$-treated mice received an injection of I $\times 10^{7} \mathrm{PFU}$ of virus into the subcutaneous antitumor on days 10, 17, or 24 after antitumor implantation. The subcutaneous antitumor volume in the treated group was significantly lower than that in the untreated mice ( $I$ vs $2: P=0.0013 ; 3$ vs $4: P=0.0045)$. The survival rate of the treated mice was significantly higher than that of the untreated mice $(I$ vs 2 : $P=0.0039 ; 3$ vs $4: P<0.000 I$ ). The survival rate of peritoneal metastasis-treated mice was significantly higher than that of peritoneal metastasis-untreated mice. (B) HFIO treatment reduced the number of abdominal antitumor nodules. HFIO $\left(\mathrm{I} \times 10^{7} \mathrm{PFU}\right)$ was injected into the subcutaneous antitumor 10 days after antitumor implantation, and the mice were sacrificed 17 days after antitumor implantation. Arrows point out metastasis antitumor nodules inside abdominal cavity. (C) HFIO decreased liver and spleen weights in liver and peritoneal metastasis models. In mice bearing liver metastases, HFIO $\left(\mathrm{I} \times 10^{7} \mathrm{PFU}\right)$ was injected into the subcutaneous antitumor $\mathrm{I} 0$ days after antitumor implantation. The mice were sacrificed 17 days after antitumor implantation. Antitumor's number in addition to liver and spleen weights were analyzed.

Abbreviations: PFU, plaque-forming unit; SD, standard deviation.

which are frequently accompanied by liver and peritoneal metastasis. Therefore, we evaluated the possibility of using HF10 to treat systemic diseases using immune-competent mice with colon cancer metastasis. Takakuwa et al ${ }^{19}$ showed that surviving mice from the HF10 treatment become resistant to the reimplantation of the antitumor, effect that is 

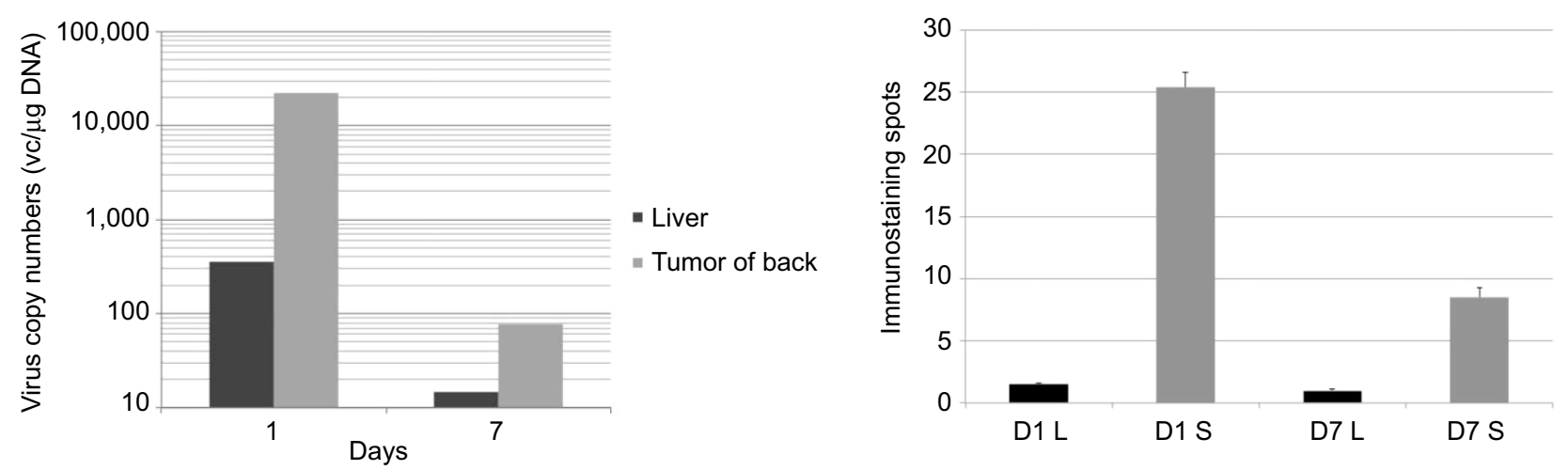

Figure 3 (A) Liver tissue contained significantly fewer HFIO DNA compared to subcutaneous antitumor tissue. DNA was extracted from liver and subcutaneous antitumors of HFIO-treated mice. Liver and subcutaneous antitumor tissues were resected on days I and 7 after HFIO administration into the subcutaneous antitumor. HFIO-specific ULI region was detected by quantitative PCR from a total DNA of $50 \mathrm{ng}$. The virus copy number was determined with standard comparison curve. (B) HFI0-immunostaining spots were significantly less abundant in liver tissue than in subcutaneous antitumor tissue. We resected liver tissue from the liver metastasis group and performed histochemical analysis with anti-HSV antibody on days I and 7 after HFI0 administration (400X).

Abbreviations: L, liver tissue; PCR, polymerase chain reaction; S, subcutaneous antitumor tissue.
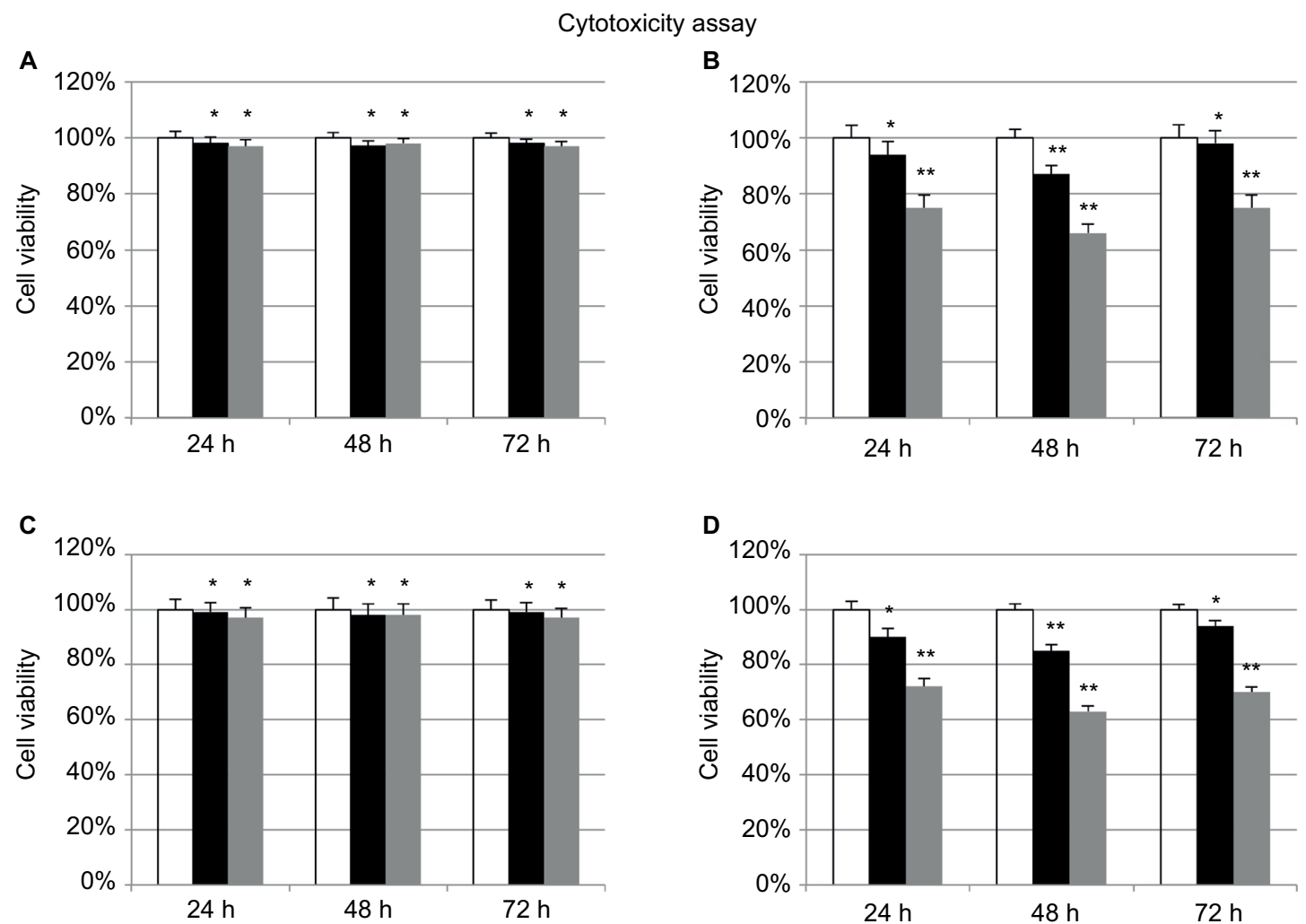

$$
\text { Control } \square \times 10 \square \times 100 \quad{ }^{*} P>0.05 \quad{ }^{* *} P>0.0001
$$

Figure 4 Whole subsets of lymphocytes from HFI0-treated mice had strong cytotoxic activity. (A) MC26 vs spleen lymphocyte (untreated). (B) MC26 vs spleen lymphocyte (treated). (C) MC26 vs thymus lymphocyte (untreated). (D) MC26 vs thymus lymphocyte (treated). We resected spleen from mice with peritoneal metastasis and thymus from mice with liver metastasis on day 13 after antitumor implantation (HFI0 was injected into the subcutaneous antitumor on day 10 after antitumor implantation) and measured lymphocytic cytotoxicity against MC26 by MTT assay.

mediated by host antitumor immunity. Consistent with this, in mice harboring antitumors on both sides of the back, an HF10 injection on one side strongly suppresses the growth of the antitumor on the other side. ${ }^{21}$ Together, these findings indicate that HF10 exerts its antitumor effects via a mecha- nism other than direct oncolysis. Similarly, immunization with HF10 (HSV-1) inhibits HSV-2 replication in the mouse vagina, reduces local inflammation, controls emergence of neurological dysfunctions of HSV-2 infection, and increases survival through host immunity. ${ }^{26}$ 

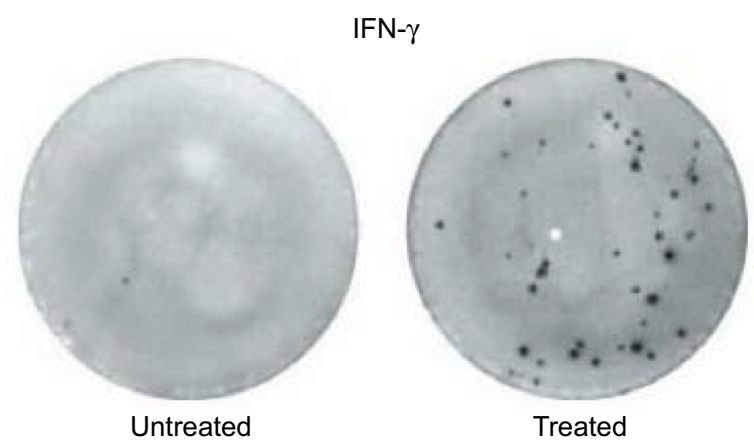

\begin{tabular}{|l|l|}
\hline Group & $\begin{array}{l}\text { Spot counts } \\
(\mathrm{n}) \pm \mathrm{SD}\end{array}$ \\
\hline Untreated & $2.40 \pm 1.6$ \\
\hline Treated & $14.0 \pm 8.0^{*}$ \\
\hline & ${ }^{*} P=0.0002$
\end{tabular}

Figure 5 IFN- $\gamma$ was predominantly expressed in whole subsets of lymphocytes from the HFI0-injected mice. We extracted whole subsets of lymphocytes from thymus of liver metastasis model mice and observed IFN- $\gamma$ expression by ELISPOT assay ( $50 \mu \mathrm{L}$ of IFN- $\gamma$ antibody and I $\times 10^{5}$ lymphocytes per well). Lymphocytes were stimulated with I $\times 10^{3} / \mathrm{mL} \mathrm{MC26}$ cells.

Abbreviations: ELISPOT, enzyme-linked immunospot; IFN- $\gamma$, interferon gamma.

In this study, we investigated these phenomena from the standpoint of the immune response. In particular, we studied the therapeutic effect of the natural HSV-1 mutant, HF10, on colorectal antitumors with liver or peritoneal metastasis. After examining the in vitro cytotoxic effect of HF10 in the mouse colon carcinoma cell line MC26, we established a diffuse liver metastasis model by splenic injection of MC26 cells and a peritoneal metastasis model by intraperitoneal injection. In both models, MC26 cells were also injected subcutaneously on the back. Using these models, we studied the therapeutic effect of HF 10 against systemic diseases by injecting virus only into the subcutaneous antitumor. Following viral injection, both the liver and peritoneal metastasis models exhibited significant inhibition of systemic antitumor growth and prolongation of survival. The effect of the oncolytic virus in the subcutaneous antitumor may depend not only on the direct cytotoxic effect of the virus but also on the host antitumor immunity. Sacrifice of the mice revealed that the virus had a curative effect on abdominal antitumors in both the liver and peritoneal metastasis models.

Furthermore, in the liver metastasis model, liver and spleen weights were significantly lower and antitumor sizes were smaller in HF10-treated mice. PCR assays on the liver tissue of the liver metastasis model and immunostaining with anti-HSV antibody revealed that the virus concentration in liver tissue was lower than the subcutaneous tumor on day 1 and also significantly lower on day 7, after HF 10 administration. Therefore, the curative effect on liver metastasis could not simply be the result of the direct oncolytic effect of the virus. Instead, as demonstrated by the lymphocyte experiments, the host antitumor immunity played a major role in the antitumor effect against systemic disease. In particular, the lymphocytes of the HF10-treated group were more cytotoxic than that of the control group. In addition, we performed ELISPOT assays using whole subsets of lymphocytes extracted from liver metastasis models and also measured the expression of IFN- $\gamma$. Whole subsets of lymphocytes of the treated group recognized antitumor-specific antigen and contained more expression spots than those of the control group.

Following HF10 treatment, viral antigens stimulate host immunity, which recognizes antitumor and viral antigens via phagocytosis by dendritic cells and natural killer cells..$^{27-30}$ This would lead to stimulation of cytotoxic CD8 T cells. On the other hand, antibodies are produced from B cells stimulated by $\mathrm{CD} 4$ helper $\mathrm{T}$ cells at least 4 weeks after first dendritic cell movement. Therefore, we believe that the results reported here reflect initial immunity mediated by cytotoxic $T$ cells. These results show that the curative effects of locally administered HF10 against systemic disease are strongly related to the host antitumor immunity, which is a different protein antigen from human. Based on our observation that HF10 can prevent metastasis, we emphasize that HF10 treatment has the potential to get developed as a novel therapy for colon cancer.

\section{Disclosure}

The authors report no conflicts of interest in this work.

\section{References}

1. Jurnal A, Siegel R, Ward E, Murray T, Xu J, Thun MJ. Cancer statistics, 2007. CA Cancer J Clin. 2007;57(1):43-66.

2. Sohn TA, Yeo CJ, Cameron JL, et al. Resected adenocarcinoma of the pancreas-616 patients: results, outcomes, and prognostic indicators. J Gastrointest Surg. 2000;4(6):567-579.

3. Lim JE, Chien MW, Earle CC. Prognostic factors following curative resection for pancreatic adenocarcinoma: a population-based, linked database analysis of 396 patients. Ann Surg. 2003;237(1):74-85.

4. Lee SR, Kim HO, Son BH, Yoo CH, Shin JH. Prognostic factors associated with long-term survival and recurrence in pancreatic adenocarcinoma. Hepatogastroenterology. 2013;60(122):358-362.

5. Riediger H, Keck T, Wellner U, et al. The lymph node ratio is the strongest prognostic factor after resection of pancreatic cancer. $J$ Gastrointest Surg. 2009;13(7):1337-1344.

6. Lorence RM, Roberts MS, O’Neil JD, et al. Phase 1 clinical experience using intravenous administration of PV701, an oncolytic Newcastle disease virus. Curr Cancer Drug Targets. 2007;7(2):169-174. 
7. Martuza RL, Malick A, Markert JM, Ruffner KL, Coen DM. Experimental therapy of human glioma by means of a genetically engineered virus mutant. Science. 1991;252(5007):854-856

8. McCart JA, Ward JM, Lee J, et al. Systemic cancer therapy with a tumorselective vaccinia virus mutant lacking thymidine kinase and vaccinia growth factor genes. Cancer Res. 2001;61(24):8751-8757.

9. Nemunaitis J, Khuri F, Ganly I, et al. Phase II trial of intratumoral administration of ONYX-015, a replication-selective adenovirus in patients with refractory head and neck cancer. J Clin Oncol. 2001;19(2):289-298.

10. Peng KW, TenEyck CJ, Galanis E, Kalli KR, Hartmann LC, Russel SJ. Intraperitoneal therapy of ovarian cancer using an engineered measles virus. Cancer Res. 2002;62(16):4656-4662.

11. Quinn C, Ma Q, Kudlac A, Palmer S, Barber B, Zhao Z. Indirect treatment comparison of talimogene laherparepvec compared with ipilimumab and vemurafenib for the treatment of patients with metastatic melanoma. Adv Ther. 2016;33(4):643-657.

12. Andtbacka RH, Ross M, Puzanov I, et al. Patterns of clinical response with talimogene laherparepvec (T-VEC) in patients with melanoma treated in the OPTiM phase III clinical trial. Ann Surg Oncol. 2016; 23(13):4169-4177.

13. Garnock-Jones KP. Talimogene laherparepvec: a review in unresectable metastatic melanoma. BioDrugs. 2016;30(5):461-468.

14. Ushijima Y, Luo C, Goshima F, Yamauchi Y, Kimura H, Nishiyama Y. Determination and analysis of the DNA sequence of highly attenuated herpes simplex virus type 1 mutant HF10, a potential oncolytic virus. Microbes Infect. 2007;9(2):142-149.

15. Tan G, Kasuya H, Sahin TT, et al. Combination therapy of oncolytic herpes simplex virus HF10 and bevacizumab against experimental model of human breast carcinoma xenograft. Int J Cancer. 2015;136(7):1718-1730.

16. Yamamura K, Kasuya H, Sahin TT, et al. Combination treatment of human pancreatic cancer xenograft models with the epidermal growth factor receptor tyrosine kinase inhibitor erlotinib and oncolytic herpes simplex virus HF10. Ann Surg Oncol. 2014;21(2):691-698.

17. Nakao A, Kasuya H, Sahin TT, et al. A phase I dose-escalation clinical trial of intraoperative direct intratumoral injection of HF10 oncolytic virus in non-resectable patients with advanced pancreatic cancer. Cancer Gene Ther. 2011;18(3):167-175.

18. Nakao A, Takeda S, Shimoyama S, et al. Clinical experiment of mutant herpes simplex virus HF10 therapy for cancer. Curr Cancer Drug Targets. 2007;7(2):169-174.
19. Takakuwa H, Goshima F, Nozawa N, et al. Oncolytic viral therapy using a spontaneously generated herpes simplex virus type 1 variant for disseminated peritoneal tumor in immunocompetent mice. Arch Virol. 2003; 148(4):813-825.

20. Kimata H, Takakuwa H, Goshima F, et al. Effective treatment of disseminated peritoneal colon cancer with new replication-competent herpes simplex virus. Hepatogastroenterology. 2003;50(52): 961-966.

21. Esaki S, Goshima F, Kimura H, Murakami S, Nishiyama Y. Enhanced antitumoral activity of oncolytic herpes simplex virus with gemcitabine using colorectal tumor models. Int J Cancer. 2013;132(7): $1592-1601$

22. Goshima F, Esaki S, Luo C, Kamakura M, Kimura H, Nishiyama Y. Oncolytic viral therapy with a combination of HF10, a herpes simplex virus type 1 variant and granulocyte-macrophage colony-stimulating factor for murine ovarian cancer. Int J Cancer. 2014;134(12): 2865-2877.

23. Fujimoto Y, Mizuno T, Sugiura S, et al. Intratumoral injection of herpes simplex virus HF10 in recurrent head and neck squamous cell carcinoma. Acta Otolaryngol. 2006;126(10):1115-1117.

24. Kimata H, Imai T, Kikumori T, et al. Pilot study of oncolytic viral therapy using mutant herpes simplex virus (HF10) against recurrent metastatic breast cancer. Ann Surg Oncol. 2006;13(8):1078-1084.

25. Kasuya $\mathrm{H}$, Kodera $\mathrm{Y}$, Nakao A, et al. Phase I dose-escalation clinical trial of HF 10 oncolytic herpes virus in 17 Japanese patients with advanced cancer. Hepatogastroenterology. 2014;61(131):599-605.

26. Luo C, Goshima F, Kamakura M, et al. Immunization with a highly attenuated replication-competent herpes simplex virus type mutant, HF 10, protects mice from genital disease caused by herpes simplex virus type 2. Front Microbiol. 2012;3:158.

27. Chiocca EA, Rabkin SD. Oncolytic viruses and their application to cancer immunotherapy. Cancer Immunol Res. 2014;2(4):295-300.

28. Woller N, Gürlevik E, Ureche CI, Schumacher A, Kühnel F. Oncolytic viruses as anticancer vaccines. Front Oncol. 2014;4:188.

29. Meshii N, Takahashi G, Okunaga S, et al. Enhancement of systemic tumor immunity for squamous cell carcinoma cells by an oncolytic herpes simplex virus. Cancer Gene Ther. 2013;20(9):493-498.

30. Shirota T, Kasuya H, Kodera Y, et al. Oncolytic herpes virus induces effective anti-cancer immunity against murine colon cancer. Hepatogastroenterology. 2011;58(110-111):1482-1489.
Oncolytic Virotherapy

\section{Publish your work in this journal}

Oncolytic Virotherapy is an international, peer-reviewed, open access online journal publishing original research, study protocols, reviews, editorials and commentaries on all aspects of oncolytic virology, namely the application of oncolytic viruses for the treatment of cancer. Specific topics in the journal include: Rationale and theoretical aspects of oncolytic virotherapy including in vitro, in vivo and mathematical

\section{Dovepress}

modeling; and practical application and problem solving in the clinic including identification of potential responders through biomarkers and genetic profiling. The manuscript management system is completely online and includes a very quick and fair peer-review system, which is all easy to use. Visit http://www.dovepress.com testimonials.php to read real quotes from published authors. 